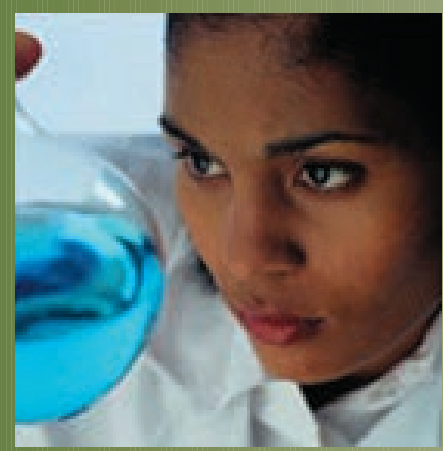

Dans tous les pays européens les femmes sont notoirement sous-représentées dans les carrières scientifiques et techniques, et cet effet est encore plus marqué aux niveaux élevés - voir par exemple le rapport She figures 2006 ${ }^{(1)}$ du Directorat Général pour la Recherche de la Commission Européenne. Les conditions économiques, sociales et culturelles varient bien sûr d'un pays à l'autre, mais il est utile de regarder comment la question est abordée ailleurs et quelles actions sont proposées pour essayer de modifier ce biais.

Les Britanniques, fidèles à leur tradition et devant respecter l'autonomie des universités, ont adopté une approche pragmatique, incitative et de longue haleine, et lancé le projet Athena - un nom choisi à dessein, la déesse à la fois sage et combative incarnant bien la philosophie du projet ! Sir David King, Government Chief Scientific Adviser, en a résumé la stratégie dans le Athena Guide to Good Practice : à court terme, inciter à ce que la proportion de femmes parmi les candidats à des postes académiques reflète leur pourcentage au niveau juste inférieur ; à moyen terme, obtenir que les recrutements et les promotions correspondent à ce pourcentage ; et faire ainsi qu'à long terme la pyramide des carrières ne soit plus biaisée.

Jean Vannimenus

\section{Le projet Athena}

Le ministre britannique de la Science ${ }^{(2)}$, Lord Sainsbury, s'était montré alarmé par les statistiques concernant la proportion de femmes professeurs dans les universités britanniques. Ce n'était pas tant la faible proportion de femmes professeurs d'université (97 sur 3092) en sciences physiques et de l'ingénieur qui l'avait surpris, mais plutôt le fait qu'en sciences de la vie moins de $10 \%$ des professeurs étaient des femmes, sachant qu'elles en constituent environ $50 \%$ de la population étudiante. C'est ce manque inacceptable de femmes aux postes élevés en science et technologie dans les universités du Royaume-Uni qui a motivé le lancement du projet Athena en février 1999.

Visant expressément à corriger ce déficit, les objectifs du projet Athena sont "d'améliorer les carrières des femmes et d'accrôitre significativement le nombre de femmes recrutées aux postes élevés, dans l'enseignement supérieur et la recherche en science et technologie ». Trois convictions majeures sous-tendent ce projet

- Le progrès de la science et de la technologie est une condition nécessaire à l'amélioration de la qualité de vie partout dans le monde.

- Il est vital que les femmes soient représentées de manière adéquate dans ce domaine, traditionnellement (et encore maintenant) dominé par le sexe masculin.

- La science ne pourra atteindre son plein potentiel que si elle peut bénéficier des talents de toute la population, et seulement à partir du moment où femmes et hommes pourront bénéficier également des opportunités qu'elle offre.

Comment y parvenir ? Cinq facteurs ont été identifiés par le projet Athena comme jouant un rôle clé dans les carrières scientifiques des femmes. Ces facteurs-clés, qui définissent ensemble une "bonne pratique » de la part des employeurs, sont :

- un bon management des personnes au sein des départements ;

- des procédures équitables de rémunération et de promotion ;

- un encouragement concret à la progression des carrières ;

- une répartition claire et affichée des rôles et responsabilités ;

- enfin, une plus grande flexibilité dans l'organisation du travail aux différentes étapes de la vie et de la carrière.

Le but d'Athena est d'encourager, développer et disséminer ces " bonnes pratiques ». Elles sont souvent simples et peu coûteuses, mais prises ensemble elles ont un impact sur le fonctionnement d'une institution. Les études menées dans le cadre d'Athena ont montré que ces «bonnes pratiques » bénéficient à tous et améliorent la qualité de la science produite. Au contraire, les "mauvaises pratiques " sont plus défavorables aux femmes à chaque étape de leur carrière, ainsi qu'à ceux qui ont des formations ou des profils de carrière non traditionnels $(3)$.

Le programme du projet Athena comporte trois phases. La première (1999-2001) a été consacrée à identifier, encourager et développer les facteurs de " bonne pratique ». À l'issue d'un concours, des petites bourses (de moins de $10000 \AA$ chacune) ont permis de récompenser 12 universités et 5 réseaux. La première année, l'objectif des bourses était de créer un réseau d'influence dans les universités et les agences gouvernementales de financement de la recherche et d'identifier les domaines où des progrès visibles pouvaient être obtenus rapidement. La deuxième année, les bourses ont été attribuées aux universités qui s'attaquaient au plus difficile objectif de changer leurs pratiques et culture. 
1 -Voir le site :

ec.europa.eu/research/sciencesociety/pdf/she_figures_ 2006_en.pdf

2 - De 1998 à novembre 2006 (NDLR).

3 - Les rapports sur les travaux effectués dans le cadre d'Athena, ainsi que le "guide de bonne pratique ", peuvent être trouvés sur le site :

www.athenaproject.org.uk.

4 - Athena est soutenue et hébergée par The Royal Society. Parmi ses autres soutiens, on peut noter British Petroleum, Institute of Physics, Pfizer, Royal Academy of Engineering, Royal Society of Chemistry, et The Wellcome Trust.
Dans la deuxième phase (2002-2003), Athena a disséminé et récompensé la "bonne pratique ». Sept universités ont été récompensées par la Royal Society pour leurs bons résultats en matière de promotion des femmes en science et technologie. En 2003, Athena a conclu ses quatre premières années de fonctionnement par une conférence majeure et par un guide de «bonne pratique $»(3)$.

Le projet Athena est maintenant dans sa phase finale (2004 à fin 2007), qui comporte deux activités principales : l'enquête ASSET ("Athena Survey of Science Engineering and Technology") et la charte Athena SWAN ("Scientific Women's Academic Network").

L'enquête ASSET comporte trois «manches», la dernière en 2006, impliquant plus de 6000 scientifiques des deux sexes travaillant dans les instituts de recherche et universités britanniques, mais aussi dans l'industrie, couvrant tous les domaines de la science et de la technologie. Les résultats seront disponibles en 2007. Les questions posées dans l'enquête s'appuyaient sur les conclusions obtenues précédemment par Athena sur les différences de perception et d'expérience des hommes et des femmes sur leur progression professionnelle. Les résultats de l'enquête montrent une convergence étonnante sur les difficultés qui limitent la progression des femmes. En particulier, dans tous les secteurs d'emploi et toutes les disciplines scientifiques, les " mauvaises pratiques » découragent les femmes à poursuivre leur carrière en science et technologie.

La charte Athena SWAN a été mise en place en 2005. À ce jour, elle a été signée par 23 universités britanniques, dont 13 ont été récompensées par une médaille de bronze ou d'argent. L'adhésion à la charte est ouverte à toute université britannique qui s'engage à contribuer à la réalisation des objectifs d'Athena. La qualité de membre de la charte permettra à une université de s'identifier comme " employeur de qualité ».

Le projet Athena a une durée limitée. Il a reçu le soutien financier de diverses agences gouvernementales et caritatives. Il emploie un faible effectif, et a bénéficié du temps et des efforts personnels d'un grand nombre de femmes (et d'hommes). En 2007, Athena travaillera avec ses partenaires à développer un ensemble d'outils qui permettront aux universités d'évaluer leur progrès en "bonne pratique ». Les partenaires (4) d'Athena s'engagent à ce que l'héritage d'Athena (incluant la charte et $A S S E T$ ) se prolonge bien au-delà de décembre 2007, date de fin du projet.

\section{Caroline Fox ${ }^{1}$ et Julia Higgins ${ }^{2}$}

1. Directrice de programme du projet ATHENA 2. Directrice de la Faculté des sciences de l'ingénieur à l'Imperial College. Ancienne présidente du Comité consultatif du projet $A T H E N A$ 